\section{The new way to bond}

Adding brackets with adhesive can be time consuming. Although the process works, why not take it to the next level and choose a more predictable, faster solution?

With the APC Flash-Free Adhesive Coated Appliance System from $3 \mathrm{M}$ Oral Care, you can revolutionise your bonding with ease.

The most advanced APC appliance system on the market, this option works so well because the nonwoven mat base adheres directly to the tooth enamel for a strong, reliable bond that eliminates the need for flash removal.

This also means no lumps of adhesive that can gather bacteria or become less aesthetic over time!

To find out more, contact the team at $3 \mathrm{M}$ today.

For more information, call 08458734066 or visit www.3m.co.uk/ apcff. $3 \mathrm{M}$ representatives remain contactable by phone or via video conferencing.

$3 \mathrm{M}$ and APC are trademarks of the $3 \mathrm{M}$ Company.

\section{Access endodontic success}

Precision-built and created in the UK, EndoPerfection's VaryFlex files offer amazing value without compromising on quality and are simple to use.

For example, the files have incredible flexibility due to the heat-treated nickel titanium alloy and a $11 \mathrm{~mm}$ shank, which is shorter than usual, making working with VaryFlex very comfortable and easy.

They can be used in much the same way as some other, popular files out there, however file creator, EndoPerfection, believe very much in the importance of ongoing training and supporting clinicians in their endeavours.

EndoPerfection is excited to announce the re-start of its face-to-face, hands-on courses around the UK: https:// endoperfection.com/products/hands-on-rotary-endo-training.

So, following the success of the online training in the last year, there are now also opportunities to enjoy this form of training.

Alongside movies demonstrating quick and easy tips for rubber dam placement and using VaryFlex Reciprocating and Taper files, there are virtual training events using conference call technology. Products are delivered to delegates in advance, so they get a great experience without having to travel.

VaryFlex is available exclusively from Wrights at https://www. wright-cottrell.co.uk/new-endoperfection-files-range/.

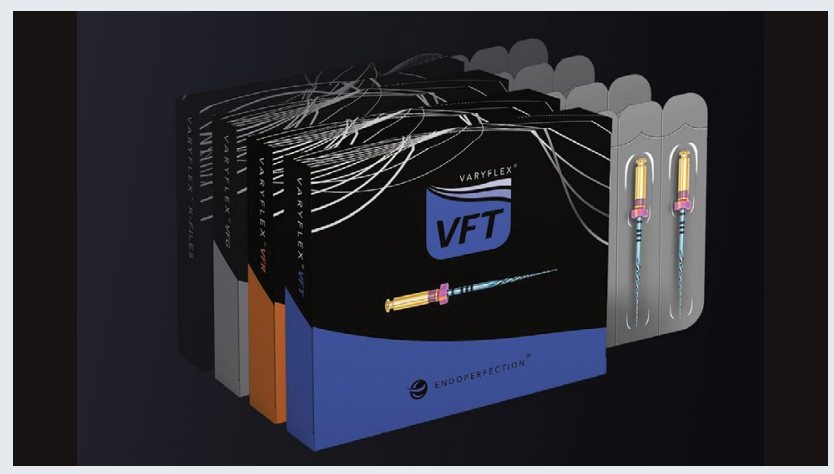

\section{Advanced infection control for your practice}

Advanced Hygenex infection control products used in hospitals and care homes are now available for dentists, schools, colleges, universities and beauty clinics.

Healthcare professionals around the globe already depend on the trusted Hygenex brand with its wide range of infection control solutions. They include:

- The UK's first $100 \%$ organic and fully biodegradable hygiene wipes that can be flushed, macerated or composted without harming the planet - Hygenex cleansing wipes pass all seven tests set by the European Disposables and Nonwovens Association (EDANA) and the International Nonwovens and Disposables Association (INDA)

- Hygenex Recoat Antimicrobial Coating System - a spray-on coating that protects surfaces for up to six months against bacteria and viruses, including SARS-CoV-2/ COVID-19

- Hand sanitiser to protect users against COVID-19 and other infections

- Medical pulp products including BioCommode, a fully self-supporting medical bedpan that needs no plastic support ... so it can be fully macerated, improving infection control.

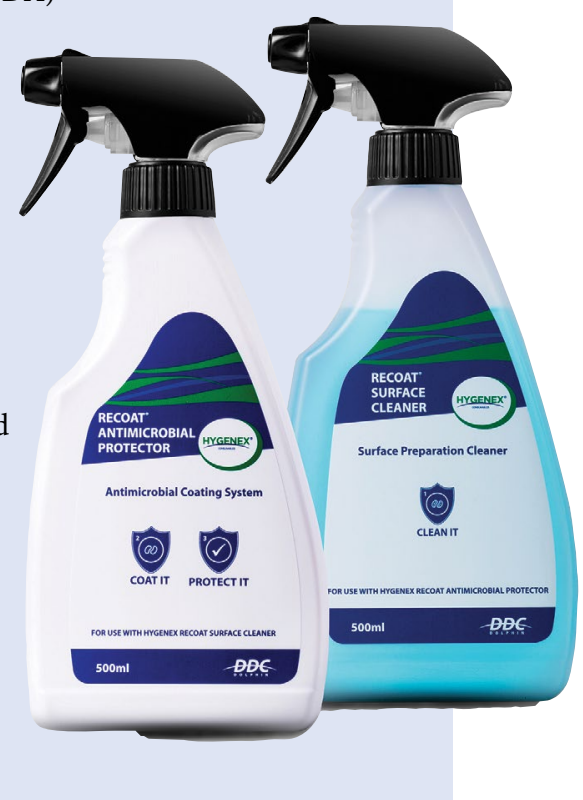

Now the Hygenex brand has been launched as a separate company in its own right, enabling a wider range of users to gain access to its infection control solutions. Dentists, schools, colleges, universities and beauty clinics will be among the first offered the new advanced products.

For more information, contact Hygenex Marketing Director Zoe Allen, 01202 731555, info@hygenex.co.uk, www.hygenex.co.uk.

\section{Superb image quality across the board}

If you provide many different types of treatment, you need an imaging solution that offers predictable, high-quality results across multiple modalities.

The CS 8200 from Carestream Dental is the system you're looking for.

Not only does this affordable, compact system offer a range of $2 \mathrm{D}$ and $3 \mathrm{D}$ imaging options, but the premium imaging functionalities and software ensure only the clearest results. This helps to ensure that you require fewer retakes and can accurately move forward no matter the challenge in front of you.

For more information, contact Carestream Dental on 0800 1699692 or visit www.carestreamdental.co.uk. 